\title{
Inverse scattering analysis of elastic half space by means of the fast volume integral equation method
}

\author{
T. Touhei \& T. Hinago \\ Department of Civil Engineering, Tokyo University of Science, Japan
}

\begin{abstract}
A method for the inverse scattering analysis for an elastic half space is developed in this article. The method is based on the fast volume integral equation developed by the author, in that the Krylov subspace is constituted by a fast method without deriving the coefficient matrix for the integral equation. The equation for the inverse scattering analysis obtained from the volume integral equation is for reconstructing fluctuations of the medium from observed scattered waves of the finite region at the free surface. The linearization for the equation is carried out by the Born approximation. The Tikhonov regularization method is also employed to have the iterative solution converge. Several numerical calculations are performed to verify the accuracy of the reconstruction of the wave field as well as the effects of the regularization method.
\end{abstract}

Keywords: inverse scattering problem, elastic half space, fast method, volume integral equation, Tikhonov regularization method.

\section{Introduction}

Identification of fluctuations of the wave field embedded in a homogeneous elastic half space from observed scattered waves is an important task in the field of earthquake engineering, seismology, and site characterization. The task for the analysis of identifying the fluctuations from the scattered waves is known as the inverse scattering analysis. There has already been vast literature concerning the inverse scattering analysis. For example, Colton and Kress [1] reported a survey of a vast number of articles on forward and inverse scattering analyses. They reported methods for acoustic and electromagnetic wave propagation based on the theory 
of operators. Colton et al. [2] presented a linear sampling method for the inverse scattering analysis of electromagnetic wave field by means of the far field operator. Fata and Guzina [3] proposed the linear sampling method by defining a near field operator to identify the scattering object hidden in an elastic half space.

In this article, a method for the inverse scattering analysis is developed by means of the fast volume integral equation method proposed by the author first $[4,5]$. The advantages of the fast volume integral equation method are in that the Krylov subspace to obtain the solution of the integral equation can be constructed by the fast algorithm without deriving the coefficient matrix. Furthermore, the volume integral shows the relationship between the fluctuation of the wave field and the scattered waves directly, which enables the direct formulation for the inverse scattering analysis. In this article, several numerical examples are presented to show the results of the inverse scattering analysis based on the formulation.

\section{Formulation for the inverse scattering analysis}

\subsection{Concept of the analysis and basic equations}

Figure 1 shows the concept of the problem defined in this article. A point source is applied to a surface of an elastic half space. The time dependency of the point source is $\exp (i \omega t)$, where $\omega$ is the circular frequency and $t$ is the time. The incident wave is propagating toward the fluctuation of the medium and scattered waves are caused due to the interaction between the scattered waves and the fluctuation of the medium. The scattered waves are observed at the finite region of the free surface. The task of this article is to reconstruct fluctuations of the medium from the observed scattered waves.

For the mathematical expression of the fluctuations of the medium, the Lamé constants and the mass density for the wave field in this article is denoted by

$$
\begin{aligned}
& \lambda(x)=\lambda_{0}+\tilde{\lambda}(x) \\
& \mu(x)=\mu_{0}+\tilde{\mu}(x) \\
& \rho(x)=\rho_{0}+\tilde{\rho}(x), \quad\left(x \in \mathbb{R}_{+}^{3}\right)
\end{aligned}
$$

where $\lambda_{0}, \mu_{0}$ and $\rho_{0}$ are the back ground Lamé constants and mass density, respectively, and $\tilde{\lambda}, \tilde{\mu}$ and $\tilde{\rho}$ are their fluctuations. Note that $x$ is the position in the elastic half space. A Cartesian coordinate system is used to describe the wave field. For example, the components of the position vector are expressed as

$$
x=\left(x_{1}, x_{2}, x_{3}\right) \in \mathbb{R}^{2} \times \mathbb{R}_{+}=\mathbb{R}_{+}^{3}
$$

where $x_{3}$ is the vertical coordinate and $x_{3}=0$ denotes the free surface of the wave field. The summation convention is applied to the subscript indices.

The governing equation used for the forward scattering problem is given by

$$
\left(L_{i j}+\delta_{i j} \rho_{0} \omega^{2}\right) u_{j}(x)=N_{i j}(x) u_{j}(x)-F_{i} \delta\left(x-x_{s}\right)
$$




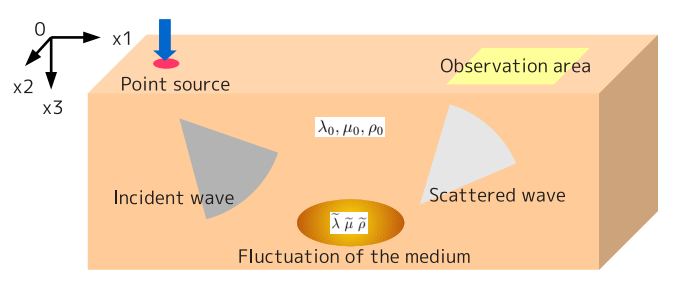

Figure 1: Concept of the analyzed model.

where $u_{i}$ is the total displacement wave field, $\delta_{i j}$ is the Kronecker delta, $\omega$ is the circular frequency, $L_{i j}$ is the operator constituted by the back ground Lamé constants such that

$$
L_{i j}=\left(\lambda_{0}+\mu_{0}\right) \partial_{i} \partial_{j}+\delta_{i j} \mu \partial_{k}^{2}
$$

$N_{i j}$ is the operator describing the fluctuation of the medium defined as

$$
\begin{aligned}
N_{i j}= & -(\tilde{\lambda}+\tilde{\mu}) \partial_{i} \partial_{j}-\delta_{i j} \tilde{\mu} \partial_{k}^{2} \\
& -\partial_{i} \tilde{\lambda} \partial_{j}-\delta_{i j} \partial_{k} \tilde{\mu}(x) \partial_{k}-\partial_{j} \tilde{\mu}(x) \partial_{i}-\delta_{i j} \tilde{\rho}(x) \omega^{2}
\end{aligned}
$$

$\delta(\cdot)$ is the Dirac function, $x_{s}$ is the position of the point source and $F_{i}$ is the amplitude of the point source. The boundary condition for the wave field is denoted by

$$
P_{i j} u_{j}=0, \quad \text { at } x_{3}=0
$$

where $P_{i j}$ is the operator given by

$$
\left[P_{i j}\right]=\left[\begin{array}{ccc}
\mu_{0} \partial_{3} & 0 & \mu_{0} \partial_{1} \\
0 & \mu_{0} \partial_{3} & \mu_{0} \partial_{2} \\
\lambda_{0} \partial_{1} & \lambda_{0} \partial_{2} & \left(\lambda_{0}+2 \mu_{0}\right) \partial_{3}
\end{array}\right]
$$

At this point, let us define the inverse scattering analysis of this article mathematically. Let $\left.v_{i}(x)\right|_{\Omega}$ be the scattered wave observed at the finite region $\Omega$ set at the free surface.

Definition 1 The inverse scattering analysis is to reconstruct $\tilde{\lambda}(x), \tilde{\mu}(x)$ and $\tilde{\rho}(x)$, $\left(x \in \mathbb{R}_{+}^{3}\right)$ from the information of $\left.v_{i}(x)\right|_{\Omega},(x \in \Omega)$, incident wave field and $G_{i j}(x, y),\left(x, y \in \mathbb{R}_{+}^{3}\right)$, where $G_{i j}(x, y)$ is the Green's function of the back ground structure of the wave field. 


\subsection{Fast volume integral equation method}

The solution of Eq. (3) together with the boundary condition shown in Eq. (7) can be expressed by the volume integral equation such that

$$
\begin{aligned}
v_{i}(x)= & -\int_{\mathbb{R}_{+}^{3}} G_{i j}(x, y) N_{j k}(y) f_{k}(y) d y \\
& -\int_{\mathbb{R}_{+}^{3}} G_{i j}(x, y) N_{j k} v_{k}(y) d y
\end{aligned}
$$

where $v_{i}$ is the scattered wave field and $f_{k}$ is the incident wave field which is given by,

$$
f_{k}(x)=G_{k j}\left(x, x_{s}\right) F_{j}
$$

and the relationship between the scattered wave field and the total wave field is

$$
u_{i}(x)=v_{i}(x)+f_{i}(x), \quad\left(x \in \mathbb{R}_{+}^{3}\right)
$$

The fast volume integral equation method employs the fast generalized Fourier and its inverse transforms, whose mathematical forms are denoted by

$$
\begin{aligned}
\left(\mathscr{U}_{i j} v_{j}\right)(\xi)= & \int_{\mathbb{R}_{+}^{3}} \Lambda_{j i}^{*}(\xi, x) v_{j}(x) d x \\
\left(\mathscr{U}_{i j}^{-1} \hat{v}_{j}\right)(x)= & \int_{\mathbb{R}^{2}} \sum_{\xi \in \sigma_{p}} \Lambda_{i j}(\xi, x) \hat{v}_{j}(\xi) d \xi_{1} d \xi_{2} \\
& +\int_{\mathbb{R}^{2}} \int_{\xi_{r}}^{\infty} \Lambda_{i j}(\xi . x) \hat{v}_{j}(\xi) d \xi_{3} d \xi_{1} d \xi_{2}
\end{aligned}
$$

where $\mathscr{U}_{i j}$ and $\mathscr{U}_{i j}^{-1}$ are the operators for the generalized Fourier and its inverse transforms, respectively, $\Lambda_{i j}$ is the kernel of the transform obtained from the following eigenvalue problem:

$$
\begin{aligned}
& L_{i j} \Lambda_{j k}(\xi, x)=-\mu_{0} \xi_{3}^{2} \Lambda_{i k}(\xi, x) \\
& P_{i j} \Lambda_{j k}(\xi, x)=0, \quad \text { at } x_{3}=0
\end{aligned}
$$

and $\xi$ is the point in the wavenumber space whose components are

$$
\xi=\left(\xi_{1}, \xi_{2}, \xi_{3}\right) \in \mathbb{R}_{+}^{3}
$$

In addition, $\xi_{r}$ in Eq. (12) is given as

$$
\xi_{r}^{2}=\xi_{1}^{2}+\xi_{2}^{2}
$$

and $\sigma_{p}$ is the subset of the wavenumber space defined by

$$
\sigma=\left\{\xi \in \mathbb{R}_{+}^{3} \mid F(\xi)=0\right\}
$$


where $F(\xi)$ is related to the Rayleigh function such that

$$
F(\xi)=\left(2 \xi_{r}^{2}-\xi_{3}\right)^{2}-4 \xi_{r}^{2} \nu \gamma
$$

Note that $\nu$ and $\gamma$ is the function of $\xi$ given as

$$
\begin{aligned}
& \nu=\sqrt{\xi_{r}^{2}-\xi_{3}^{2}} \\
& \gamma=\sqrt{\xi_{r}^{2}-\xi_{3}^{2}\left(c_{T} / c_{L}\right)^{2}}
\end{aligned}
$$

where $c_{T}$ and $c_{L}$ is the $\mathrm{S}$ and $\mathrm{P}$ wave velocities, respectively.

The application of the generalized Fourier transform to the volume integral equation shown in Eq. (9) leads to

$$
\begin{aligned}
\hat{v}_{i}(\xi)= & -\hat{h}(\xi) \mathscr{U}_{i j} N_{j k} f_{k} \\
& -\hat{h}(\xi) \mathscr{U}_{i j} N_{j k} \mathscr{U}_{k l}^{-1} \hat{v}_{l}(\xi)
\end{aligned}
$$

where

$$
\hat{h}(\xi)=\frac{1}{\mu_{0} \xi_{3}^{2}-\rho_{0} \omega^{2}+i \epsilon}
$$

and $\hat{v}$ is the generalized Fourier transform of $v$. Note that $\epsilon$ in Eq. (20) is the infinitesimally small positive real number. Equation (19) can be solved by means of the Krylov subspace method. During the iterative procedure, it is possible to introduce the fast algorithm for the generalized Fourier transform. As a result, a fast method for the volume integral equation method can be established for the forward scattering analysis.

\subsection{Method for the inverse scattering analysis}

We are in a stage for the formulation of the inverse scattering analysis. It is not very difficult to rewrite Eq. (9) into the equation that describes the relationship between the fluctuation of the wave field and scattered waves, which is as follows:

$$
\begin{aligned}
v_{i}(x)= & -\int_{\mathbb{R}_{+}^{3}} G_{i j}(x, y) M_{j k}\left(f_{1}, f_{2}, f_{3}, y\right) q_{k}(y) d y \\
& -\int_{\mathbb{R}_{+}^{3}} G_{i j}(x, y) M_{j k}\left(v_{1}, v_{2}, v_{3}, y\right) q_{k}(y) d y
\end{aligned}
$$

where $q_{k}$ is the states vector describing the fluctuation of the wave field such that:

$$
\left(q_{k}(y)\right)=(\tilde{\lambda}(y), \tilde{\mu}(y), \tilde{\rho}(y)), \quad\left(y \in \mathbb{R}_{+}^{3}\right)
$$

and $M_{j k}$ is the operator defined as

$$
\begin{aligned}
& M_{j 1}\left(f_{1}, f_{2}, f_{3}, y\right)=-\partial_{j} \partial_{k} f_{k}-\partial_{k} f_{k} \partial_{j} \\
& M_{j 2}\left(f_{1}, f_{2}, f_{3}, y\right)=-\partial_{j} \partial_{k} f_{k}-\partial_{k}^{2} f_{j} \partial_{j}-\partial_{k} f_{j} \partial_{k}-\partial_{j} f_{k} \partial_{k} \\
& M_{j 3}\left(f_{1}, f_{2}, f_{3}, y\right)=-\omega^{2} f_{j}
\end{aligned}
$$


The explicit form of $M_{j k}\left(v_{1}, v_{2}, v_{3}, y\right)$ would be clear from Eq. (23). Note that Eq. (21) is the non-linear equation in terms of $q_{k}$, since $M_{j k}\left(v_{1}, v_{2}, v_{3}, y\right)$ depends on $q_{k}$. Therefore, for simplicity, the Born approximation is applied to Eq. (21) to linearize the equation for the inverse scattering analysis. Furthermore, the equation for the inverse scattering analysis should be the relationship between the fluctuation of the medium and the observed scattered waves. As a result, the equation for the inverse scattering analysis becomes as

$$
\left.v_{i}(x)\right|_{\Omega}=-\chi_{\Omega}(x) \int_{\mathbb{R}_{+}^{3}} G_{i j}(x, y) M_{j k}\left(f_{1}, f_{2}, f_{3}, y\right) q_{k}(y) d y
$$

where $\chi_{\Omega}$ is the characteristic function defined by

$$
\chi_{\Omega}(x)= \begin{cases}1 & x \in \Omega \\ 0 & x \notin \Omega\end{cases}
$$

To solve Eq. (24) without deriving the coefficient matrix, the generalized Fourier and its inverse transforms are applied to this equation. The result is

$$
\left.v_{i}(x)\right|_{\Omega}=-\chi_{\Omega}(x) \mathscr{U}_{i j}^{-1} \hat{h}(\xi) \mathscr{U}_{j k} M_{k l}\left(f_{1}, f_{2}, f_{3}, x\right) q_{l}(x)
$$

It may be true that Eq. (26) is the equation for the inverse scattering analysis to which the fast method is applicable, there is, however, a problem in the convergence of the solution in the case that Krylov subspace iteration method is employed [6]. In order to resolve the problem of the convergence of the solution, the Tikhonov regularization method [1] is applied to Eq. (26). The form of the equation to which the Tikhonov regularzization method is applied is as follows,

$$
\left.A_{i j}^{*} v_{j}(x)\right|_{\Omega}=\left(\alpha \delta_{i j}+A_{i k}^{*} A_{k j}\right) q_{j}(x)
$$

where $\alpha$ is the regularization parameter, $A_{i j}$ is the operator given as

$$
A_{i j}=-\chi_{\Omega}(x) \mathscr{U}_{i j}^{-1} \hat{h}(\xi) \mathscr{U}_{j k} M_{k l}\left(f_{1}, f_{2}, f_{3}, x\right)
$$

and $A_{i j}^{*}$ is the adjoint operator for $A_{i j}$ satisfying the following equation:

$$
\left(\varphi_{i}(x), A_{i j} q_{j}\right)_{\Omega}=\left(A_{j i}^{*} \varphi_{i}(x), q_{j}\right)_{\mathbb{R}_{+}^{3}}
$$

Note that $(\cdot, \cdot)_{\Omega}$ and $(\cdot, \cdot)_{\mathbb{R}_{+}^{3}}$ is respectively defined as

$$
\begin{gathered}
(f, g)_{\Omega}=\int_{\Omega} f^{*}(x) g(x) d x_{1} d x_{2}, \quad f, g \in L_{2}(\Omega) \\
(f, g)_{\mathbb{R}_{+}^{3}}=\int_{\mathbb{R}_{+}^{3}} f^{*}(x) g(x) d x_{1} d x_{2} d x_{3}, \quad f, g \in L_{2}\left(\mathbb{R}_{+}^{3}\right)
\end{gathered}
$$

In the following numerical examples, Eq. (27) is used for the inverse scattering analysis. 


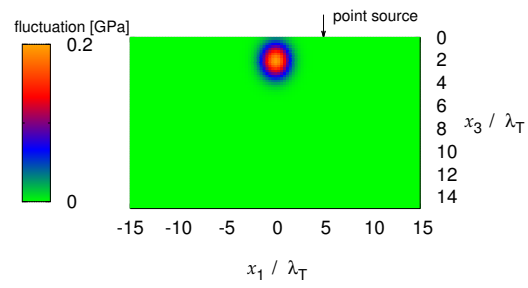

Figure 2: Target model for the inverse scattering analysis.

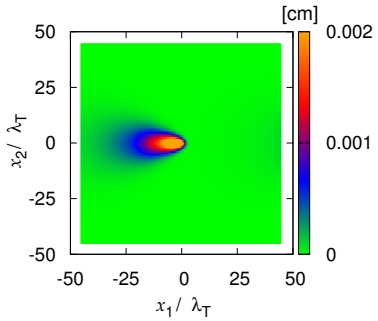

(a) at the free surface

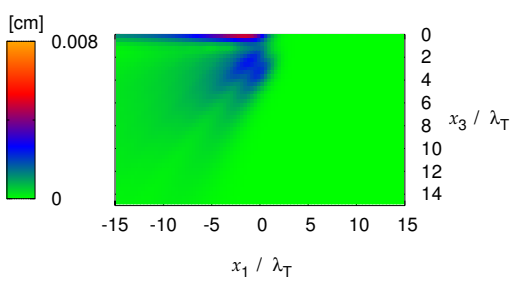

(b) at the vertical plane

Figure 3: Results of the forward scattering analysis. Amplitudes of the scattered waves are shown.

\section{Numerical examples}

\subsection{Target model}

Results of the forward scattering analysis is necessary to carry out the inverse scattering analysis. The target model is shown in Fig. 2, in which the fluctuation of the wave field and the location of the point source is shown. In Fig. 2, the fluctuation of the Lamè constants $\tilde{\lambda}$ and $\tilde{\mu}$ is shown. The fluctuation of the mass density is set here by $\tilde{\rho}=0$. In addition, the back ground Lamé constants are $\lambda_{0}=4 \mathrm{GPa}$ and $\mu_{0}=2 \mathrm{GPa}$ and the back ground mass density is $\rho_{0}=2 \mathrm{~g} / \mathrm{cm}^{3}$ for the target model. The amplitude of the excitation force is $1.0 \times 10^{10} \mathrm{~N}$, whose direction is vertical with the frequency of $1 \mathrm{~Hz}$. Therefore the wavelength of $\mathrm{S}$ and $\mathrm{P}$ waves are $1 \mathrm{~km}$ and $2 \mathrm{~km}$ respectively. In Fig. $2, \lambda_{T}$ denotes the $\mathrm{S}$ wavelength. The number and the interval of grids to discretize the integral equation $n_{j}=512$ and $\Delta x_{j}=0.176 \mathrm{~km}$, where $n_{j}$ and $\Delta x_{j},(j=1,2,3)$ denotes the number of grids and the interval of grids in the $-t h$ component.

Figure 3 shows the amplitude of the scattered waves obtained from the forward scattering analysis. The amplitudes of the waves are shown at the free surface as well as at the vertical plane. It is found from Fig. 3(a) that the large amplitude of 


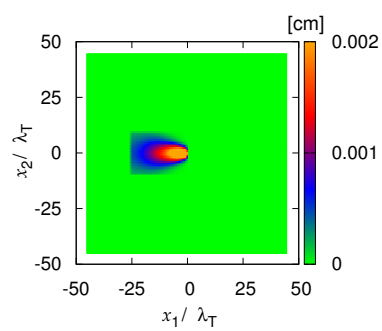

Figure 4: Picked up data of the scattered waves at the free surface.

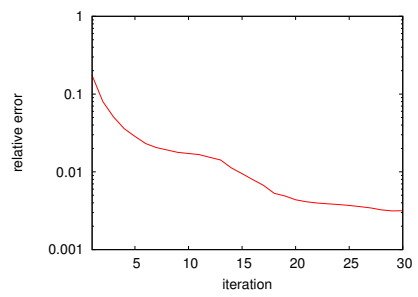

Figure 5: Convergence properties of the solution by Bi-CGSTAB method.

the waves can be observed from just above the fluctuation. The forward scattering is also found to be outstanding. According to the Fig. 3(b), the high amplitude areas can be recognized along the free surface and toward the downward direction in the forward side. These high amplitude areas are corresponding to the Rayleigh wave and the body waves. The results of the forward scattering analysis are found to well explain the phenomena of wave propagation in an elastic half space.

\subsection{Inverse scattering analysis}

Based on the results of the forward scattering analysis, the fluctuation of the wave field is reconstructed. The picked up data of scattered wave used to reconstruct the fluctuation is shown in Fig. 4.

The Bi-CGSTAB method is employed to solve Eq. (27). Figure 5 shows the convergence properties of the solution of Eq. (27) in the case that $\alpha=1.0 \times 10^{-6}$. The horizontal axis of Fig. 5 is the number of iterations and the vertical axis is the relative error. It is found from Fig. 5 that the convergence of the solution becomes slower as the iteration number increases. The following results of the reconstruction of the fluctuation are at the iteration number of thirty. The relative error at this iteration number is about 0.003 .

Figures 6 and 7 show the comparisons of the target and the results of the reconstruction of $\tilde{\mu}$ and $\tilde{\lambda}$ at the depth of $x_{3} / \lambda_{T}=2$, respectively. It is found from Figs. 6 and 7 that the reconstructed amplitudes of the fluctuations are almost 


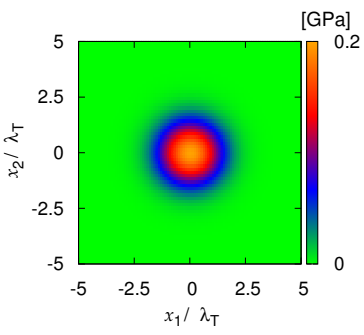

(a) target for $\tilde{\mu}$.

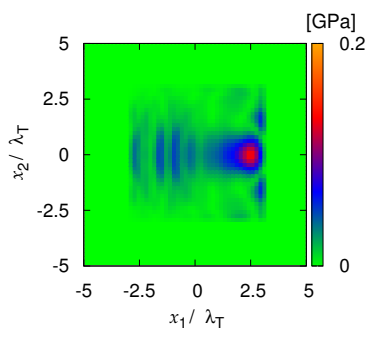

(b) reconstruction of $\tilde{\mu}$.

Figure 6: Results of the reconstruction of $\tilde{\mu}$ at the depth of $x_{3} / \lambda_{T}=2$.

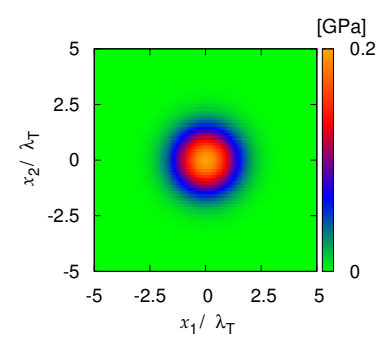

(a) target for $\tilde{\lambda}$.

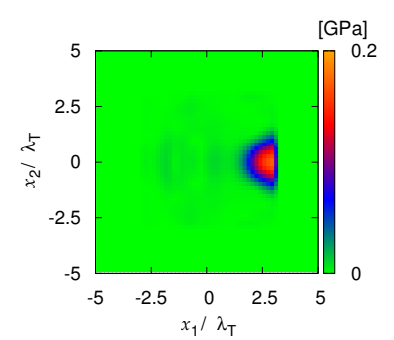

(b) reconstruction of $\tilde{\lambda}$.

Figure 7: Results of the reconstruction of $\tilde{\lambda}$ at the depth of $x_{3} / \lambda_{T}=2$.

equal to the targets. The spatial spreads of the reconstructed results are, however, different from the targets. The reconstructed results are shifted to the right direction when compared to the targets. Careful investigations are required to explain the reason for the discrepancies between the reconstructions and targets. It can be said, however, one of the reasons for this is due to the location of the excitation force at the free surface that is applied at the right side of the fluctuation. Improvement of accuracy of spatial spreads of the fluctuation is the task of the future. The processor used for the analysis is AMD Opteron 6220. The real time required for the computation was $840 \mathrm{~min}$, in the case that the four core MPI parallel processing was used.

\section{Conclusion}

In this article, the fast method for the inverse scattering analysis of elastic half space was developed by means of the volume integral equation method. Observed scattered waves at the free surface of a restricted region were used to reconstruct fluctuations of the wave field. The Tikhonov regularization method 
was employed to have the solution of the Born approximated integral equation converge. According to the numerical results for the inverse scattering analysis, the reconstructed fluctuations were found to have almost the same amplitudes with the target model. The accuracy of the reconstruction of the spatial spread of the fluctuations, however, should be improved. This improvement is the task for the future.

\section{References}

[1] Colton, D. and Kress, R.: Inverse Acoustic and Electromagnetic Scattering Theory, Springer, Berlin, 1998.

[2] Colton, D, Haddar, H. and Piana, M.: The linear sampling method in inverse electromagnetic scattering theory, Inverse Problems, Vol. 19, pp. S105-S137, 2003.

[3] Fata, S.N. and Guzina, B.B.: The linear sampling method for near-field inverse problems in elastodynamics, Inverse Problems, Vol. 20, pp. 713-736, 2004.

[4] Touhei, T.: Generalized Fourier transform and its application to the volume integral equation for elastic wave propagation in a half space, International Journal of Solids and Structures, Vol. 46, pp. 52-73, 2009.

[5] Touhei, T.: A fast volume integral equation method for elastic wave propagation in a half space, International Journal of Solids and Structures, Vol. 48, pp. 3194-3208, 2011.

[6] Touhei, T., Takagishi, T., Wajima, Y. and Kuranami, K.: Inverse scattering analysis of an elastic half space based on a fast volume integral equation method, Boundary Elements and other Mesh Reduction Methods, Vol. 32, pp. 111-121, 2010. 